\title{
Which factors predict outcomes of neonates with hypoxic-ischemic encephalopathy following therapeutic hypothermia?
}

\author{
Gyu Hong Shim, MD, PhD \\ Department of Pediatrics, Inje University Sanggye Paik Hospital, Inje University College of Medicine, Seoul, Korea
}

\section{Key message}

Determining the therapeutic hypothermia and predict long-term prognosis quickly and accurately in infants with moderate to severe hypoxic-ischemic encephalopathy requires a thorough history taking, physical examination, amplitude-integrated electroencephalography, brain magnetic resonance imaging with diffusion-weighted imaging and proton magnetic resonance spectroscopy, heart evaluation (cardiac enzymes, electrocardiography, and echocardiography), and several other biomarkers.

Hypoxic-ischemic encephalopathy (HIE), which develops after perinatal asphyxia, is a leading cause of death or long-term neurological impairment in infants born at term or near term. Around 20\%-50\% of infants with HIE die early in infancy, and $25 \%-60 \%$ of surviving infants have long-term neurological disorders such as cerebral palsy, epilepsy, intellectual disability, and learning disabilities. ${ }^{1)}$ Studies of therapeutic hypothermia (TH), the current standard therapy $\left(33^{\circ} \mathrm{C}-34^{\circ} \mathrm{C}\right.$ for 72 hours within 6 hours of birth), decreased mortality with relative risk (RR) of 0.75 (95\% confidence interval [CI], 0.64-0.88; risk difference [RD], -9\%), and neurodevelopmental disability in survivors with RR of 0.67 (95\% CI, 0.55-0.80; RD, -13\%) in infants $\geq 35$ weeks' gestational age with moderate to severe HIE. ${ }^{2,3)} \mathrm{TH}$ also decreased the neuromotor delay (Bayley Scales of Infant Development - Mental Development Index, more than 2 standard deviation (SD) below the mean; RR, 0.75; 95\% CI, 0.59-0.94; RD, -9\%) and developmental delay (Bayley Scales of Infant Development - Mental Development Index, more than 2 SD below the mean; RR, 0.74; 95\% CI, 0.58-0.94; RD, -10\%) in survivors and decreased the risk of cerebral palsy in survivors with RR of 0.66 (95\% CI, 0.64-0.88; RD, -12\%). ${ }^{3)}$

Identifying the early predictive factors for mortality and neurological prognosis in these HIE infants is particularly important in predicting relevant clinical outcomes and making rapid rational clinical decisions. Therefore, studies have examined various factors for predicting the severity and prognosis of infants with HIE (Table 1). The ideal predictors for these predictions in HIE infants should be sensitive, specific, early, quick, and easy to per- form. In the medical history and physical examinations, low 1and 5-min Apgar scores, the need for advanced neonatal resuscitation, and abnormal neurological examination findings are predictors of the need for TH and poor prognosis in infants with HIE. ${ }^{2)}$ Amplitude-integrated electroencephalography (aEEG) or conventional multichannel EEG performed within the first 7 days after birth of HIE infants undergoing TH is known to play a role as a neurophysiological predictive test. ${ }^{4)} \mathrm{A}$ brain magnetic resonance imaging (MRI) scan is conducted to determine the extent and degree of brain injury in infants with HIE. ${ }^{3,4)}$ Many additional tests, such as diffusion-weighted imaging (DWI), diffusion-tensor imaging, and proton magnetic resonance spectroscopy ( $\left.{ }^{1} \mathrm{H}-\mathrm{MRS}\right)$, have recently been used. ${ }^{3-5)}$ Ouwehand et al. ${ }^{4)}$ performed a meta-analysis of the predictors of outcomes in HIE following hypothermia using 37 articles on aEEG, MRI, DWI, and ${ }^{1} \mathrm{H}-\mathrm{MRS}$. This study showed that abnormal aEEG findings at 36 hours (diagnostic odds ratio [DOR], 62.7; 95\% CI, 19.5-202.0; $P<0.001$ ), injury to the posterior limb of the internal capsule on MRI (DOR, 39.5; 95\% CI, 16.9-92.3; $P<0.001$ ) or the thalami on DWI (DOR, 50.2; 95\% CI, 19.1131.7; $P<0.001)$, and an increased lactate $/ \mathrm{N}$-acetylaspartate peak on ${ }^{1} \mathrm{H}-\mathrm{MRS}$ within 7 days (DOR, 64.8; 95\% CI, 28.8$145.9 ; P<0.001)$ are strong predictors of adverse neurodevelopmental outcomes.

The American College of Obstetricians and Gynecologists guideline recommends using brain MRI 24-96 hours after birth to describe the timing of brain injury in HIE infants; conversely, it recommends using MRI 10 days after birth to describe the extent of brain injury. ${ }^{5)}$ Some studies have suggested that additional tests, such as near-infrared spectroscopy and somatosensory-evoked potentials, could also be used as predictive factors. ${ }^{2,4)}$ Bhasin and Kohli') ${ }^{6}$ suggested that elevated cardiac enzymes, such as serum creatine kinase (CK)-T $(P<0.001)$, CK-MB $(P<0.001)$, and troponin T $(P=0.002)$; abnormal electrocardiography findings $(P<0.001)$; and a decreased left ventricular ejection fraction and right ventricular ejection fraction on echocardiography $(P<$ 0.001 ), were associated with mortality and severity in infants

Corresponding author: Gyu Hong Shim, MD, PhD, Department of Pediatrics, Inje University Sanggye Paik Hospital, Inje University College of Medicine, 1342 Dongil-ro, Nowon-gu, Seoul 01757, Korea 
Table 1. Predictive factors of neurodevelopmental outcomes and mortality in neonates with hypoxic-ischemic encephalopathy

\begin{tabular}{|c|c|}
\hline Category & Predictive factors \\
\hline \multirow[t]{3}{*}{ History and physical examinations } & 1 or 5-min Apgar scores \\
\hline & Neonatal resuscitation level \\
\hline & Early neurologic examination \\
\hline \multirow[t]{2}{*}{ EEG } & Single- or 2-channel aEEG \\
\hline & Multichannel EEG \\
\hline \multirow[t]{4}{*}{ Brain imaging } & Conventional brain MRI \\
\hline & DWI or DTI \\
\hline & ${ }^{1} \mathrm{HMRS}$ \\
\hline & Cranial US \\
\hline \multicolumn{2}{|l|}{ Near-infrared spectroscopy } \\
\hline \multicolumn{2}{|l|}{ Somatosensory-evoked potentials } \\
\hline \multirow[t]{3}{*}{ Cardiac evaluation } & ECG: heart rate variability \\
\hline & Echocardiography: LVEF, RVEF \\
\hline & Cardiac enzymes: CK, CK-MB, CK-BB, Troponin-I, Troponin-T \\
\hline \multirow[t]{5}{*}{ Other biomarkers } & Umbilical artery and vein $\mathrm{pH}$, base excess \\
\hline & ALT, LDH, uric acid, lactate, activin A, NSE, myelin basic protein, protein S-100, glial fibrillary acidic protein, UCH-L1, pNF-H \\
\hline & Serum and urinary L/C ratio \\
\hline & Serum IL-1, IL-1ß, IL-6, IL-16 \\
\hline & CSF NSE and IL-1 $\beta$ \\
\hline
\end{tabular}

EEG, electroencephalography; aEEG, amplitude-integrated EEG; MRI, magnetic resonance imaging; DWI, diffusion-weighted imaging; DTI, diffusion-tensor imaging; ${ }^{1} \mathrm{H}$ MRS, proton magnetic resonance spectroscopy; US, ultrasonography; ECG, electrocardiography; LVEF, left ventricular ejection fraction; RVEF, right ventricular ejection fraction; CK, creatine kinase; ALT, alanine aminotransferase; LDH, lactate dehydrogenase; NSE, neuron-specific enolase; UCH-L1, ubiquitin carboxy-terminal hydrolase L1; pNF-H, phosphorylated axonal form of the neurofilament subunit NF-H; L/C, lactate/ creatinine; IL, interleukin; CSF, cerebrospinal fluid.

with HIE. In addition, some studies have shown that decreased heart rate variability has the potential for severity assessment and long-term prognosis predictions in infants with HIE. ${ }^{2)}$ In HIE infants, increased serum CK-BB and urine lactate/creatinine ratios were observed at disease onset, making them useful predictors of HIE. ${ }^{1)}$ In an umbilical blood gas analysis, a $\mathrm{pH}<7.00$ indicates a $50 \%$ chance of an abnormal outcome; however, its positive predictive value for moderate to severe HIE is low. ${ }^{2)}$ Additionally, elevated levels of serum protein S100, glial fibrillary acid protein, ubiquitin carboxy-terminal hydrolase L1, interleukin (IL)-6, IL-16, Activin A, and cerebrospinal fluid neuronspecific enolase and IL-1 $\beta$ were significantly associated with abnormal outcomes in survivors. $1,2,6,7)$

Sabahi et al. ${ }^{8)}$ showed that the need for advanced neonatal resuscitation (odds ratio [OR], 23.55; 95\% CI, 2.3-238.6; $P=$ 0.0075 ) was an independent predictive factor of death, while severely abnormal aEEG findings (OR, 63.0; 95\% CI, 7.9-504.6; $P=0.0001$ ) were independent predictive factors of severity in infants with HIE. The combination of biomarkers and neonatal resuscitation level may provide a rapid and accurate method of predicting moderate to severe HIE., ${ }^{2,910)}$ Among them, aEEG is a widely used bedside tool for identifying potential candidates for $\mathrm{TH}$ among infants with HIE, and it is known that persistently abnormal aEEG results beyond 48 hours after birth (DOR, 66.9; 95\% CI, 19.7-227.2) are associated with poor long-term prognosis as reported by Sabahi et al. ${ }^{8,10)}$

In conclusion, to determine $\mathrm{TH}$ and predict the long-term prognosis quickly and accurately in infants with HIE, it is necessary to make comprehensive judgments using medical history, physical examination, EEG, brain imaging, cardiac evaluation, and several biomarkers.

\section{Conflicts of Interest}

No potential conflicts of interest relevant to this article are reported.

See the article "Predictive factors of death in neonates with hypoxic ischemic encephalopathy receiving selective head cooling” at https://doi.org/10.3345/cep.2019.01382.

\section{References}

1. Alkholy UM, Abdalmonem N, Zaki A, Ali YF, Mohamed SA, Abdelsalam NI, et al. Early predictors of brain damage in full-term newborns with hypoxic ischemic encephalopathy. Neuropsychiatr Dis Treat. Neuropsychiatr Dis Treat 2017;13:2133-9.

2. Ahearne CE, Boylan GB, Murray DM. Short and long term prognosis in perinatal asphyxia: an update. World J Clin Pediatr 2016;5:67-74.

3. Jacobs SE, Berg M, Hunt R, Tarnow-Mordi WO, Inder TE, Davis PG. Cooling for newborns with hypoxic ischaemic encephalopathy. Cochrane Database Syst Rev 2013;1:CD003311.

4. Ouwehand S, Smidt LCA, Dudink J, Benders MJNL, de Vries LS, Groenendaal F, et al. Predictors of outcomes in hypoxic-ischemic encephalopathy following hypothermia: a meta-analysis. Neonatology 2020;117: 411-27.

5. D’Alton GD, Berkowitz RL, Bienstock J, Ghidini A, Goldsmith J, Higgins $\mathrm{R}$, et al. Executive summary: neonatal encephalopathy and neurologic outcome, second edition - report of the American College of Obstetricians and Gynecologists' Task Force on Neonatal Encephalopathy. Obstet 
Gynecol 2014;123:896-901.

6. Bhasin $\mathrm{H}$, Kohli C. Myocardial dysfunction as a predictor of the severity and mortality of hypoxic ischaemic encephalopathy in severe perinatal asphyxia: a case-control study. Paediatr Int Child Health 2019;39:259-64.

7. Florio P, Abella R, Marinoni E, Di Iorio R, Li Volti G, Galvano F, et al. Biochemical markers of perinatal brain damage. Front Biosci (Schol Ed) 2010;2:47-72.

8. Basiri B, Sabzehei M, Sabahi M. Predictive factors of death in neonates with hypoxic-ischemic encephalopathy receiving selective head cooling. Clin Exp Pediatr 2021;64:180-7. https://doi.org/10.3345/cep.2019. 01382.

9. van Laerhoven $H$, de Haan TR, Offringa M, Post B, van der Lee JH. Prognostic tests in term neonates with hypoxic-ischemic encephalopathy: a systematic review. Pediatrics 2013;131:88-98.

10. Chandrasekaran M, Chaban B, Montaldo P, Thayyil S. Predictive value of amplitude-integrated EEG ( $\mathrm{aEEG})$ after rescue hypothermic neuroprotection for hypoxic ischemic encephalopathy: a meta-analysis. J Perinatol 2017;37:684-9.

How to cite this article: Shim GH. Which factors predict outcomes of neonates with hypoxic-ischemic encephalopathy following therapeutic hypothermia? Clin Exp Pediatr 2021;64: 169-71. https://doi.org/10.3345/cep.2020.01459 